\title{
Choreographic Performance Systems
}

\author{
Johannes Birringer
}

\section{Digital Performance}

Around the turn of the millennium reviewers noted that the marriage of dance and technology had produced a few significant works which startled audiences and shifted attention to what we now call digital performance. While the growth of computer-based art is an accepted phenomenon in globalized technological cultures, the genre of digital performance is still adolescent and thus in need of historical and conceptual underpinnings. ${ }^{1}$ The more sustained lineage of dance on screen and multimedia performances which incorporate projections of screen images offers a background for understanding the compatibility between live dance and the moving image, yet the incursion of software into choreographic working process is a different matter.

Digital performance, to begin, is not a screen-based medium. Rather, it is characterized by an interface structure and computational processes that are integral for composition, evolving content, aesthetic techniques, interactive configurations and delivery forms. In many instances, the integration of human-machine interfaces implies the design of interactive systems, with 3D motion sensing set ups (for example Kinect) or wearable instruments that control real-time synthesis of digital outputs. Installation architectures compete with the stage - contextual design of programmable systems becomes a new form of architecture, protocol, and bio-informatic space. In the following, I review the historical development of video-dance, motion capture, and the dance \& 
technology movement of past decades, before addressing system design and its role in the choreographic organization of real-time interactive dance.

When widely known choreographers Merce Cunningham and Bill T. Jones collaborated with digital artists and computer scientists (Paul Kaiser, Shelley Eshkar, Michael Girard, Marc Downie) to create a series of dance works and installations exploring the artistic potential of motion capture technology - Hand-drawn Spaces (1998), BIPED (1999), Ghostcatching (1999), Loops (2001-2004) - reviews in Time Magazine spoke of 'hypnotic groundbreaking performances' bringing dance, the most physical of the arts, into the digital age. But motion capture-based digital graphics had

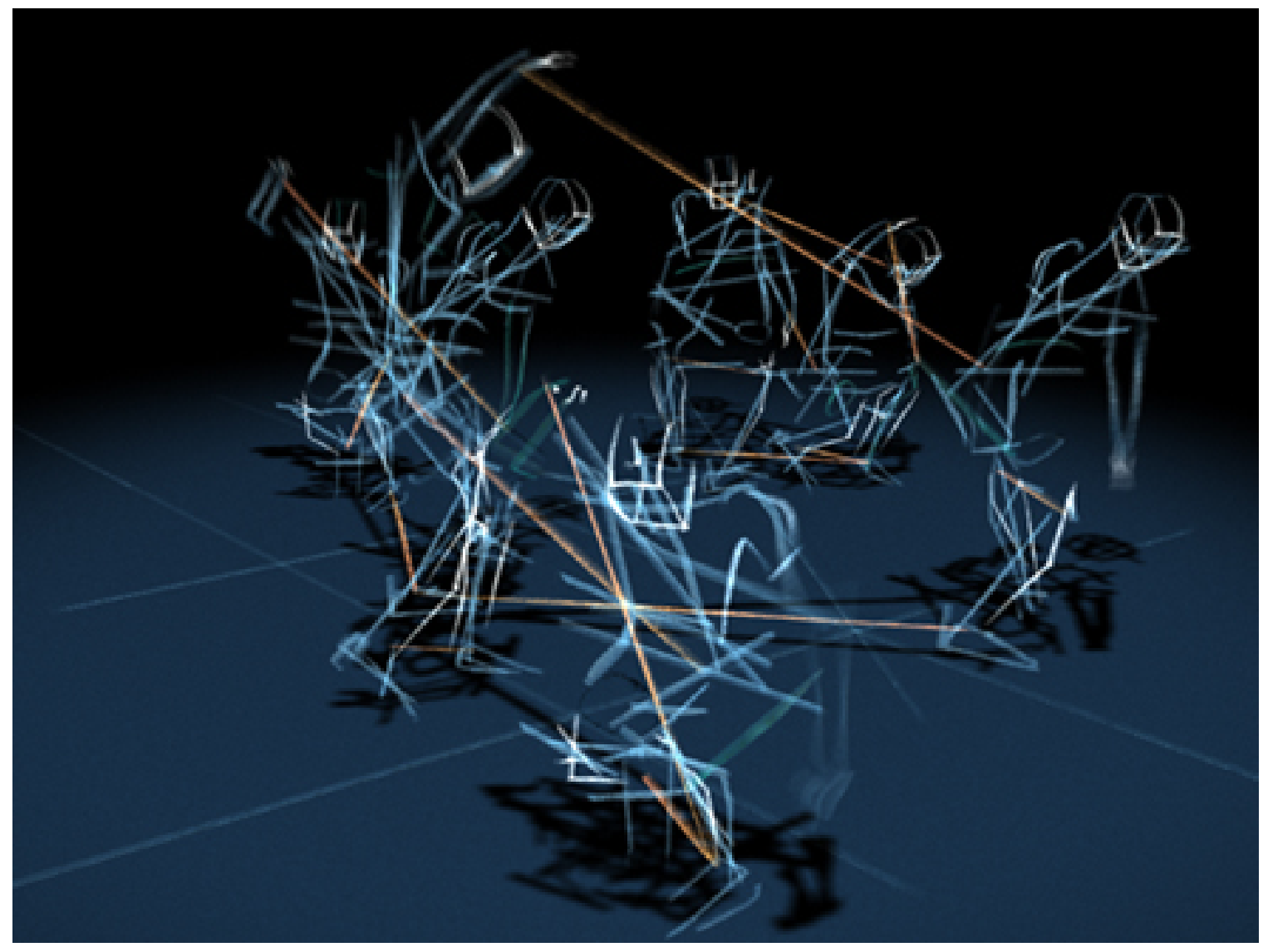

Photograph 30.1 Bill T. Jones, with Paul Kaiser/Shelley Eshkar in Ghostcatching (1999). Photo courtesy of Paul Kaiser. 
already been widely seen in Hollywood and Hong-Kong martial arts movies, and now we await the refinements of 3D Cinema. Wim Wenders released his film homage to Pina Bausch - Pina: Dance Or We Are Lost - in 3D in 2011. When I went to see Wayne McGregor's live dance Atomos at London’s Sadler’s Wells (2014), I was also handed 3D glasses at the door. Digital animation is a staple of the film industry, television advertising, MTV, club-house VJ'ing, and games design. Tight choreographic operations mixing live and prerecorded video projections are commonplace at rock concerts. The question of what is groundbreaking in the coupling of dance and technology, therefore, must be examined carefully before we make rash claims.

\section{Kinetic Camera Connections}

Video cameras play a dramaturgic role in theatre productions. Some directors (Frank Castorf, Katie Mitchell, Robert Lepage and so on) have perfected the use of onstage camera crews, as did the Wooster Group, Rimini Protokoll, the Builders Association and others in their intermedial performances. The same tendency to 'audio-visualize' music theatre and composition is seen in contemporary opera and sonic art. William Kentridge’s animated drawings/films for opera productions (The Nose; Lulu) are fascinating examples of projection techniques using layering and silhouettes. Kentridge also worked with Handspring Puppet Company - I am tempted to think that the use of video animation in theatre belongs to the conceptual tradition of the Übermarionette (Kleist). Yet the role of the physical body and the limits of physical presence vis à vis camera and computer software have become important theoretical issues for discussions of technological embodiment, not to speak of virtual realms of cybertheatre (Giannachi 2004; Parker- 
Starbuck 2011). Immersive techniques of improvisation inside systems - and how their kinaesthetic experience affects us - constitute major artistic concern. It is important for the field to worry about integrating such expanded notions of choreography and software design/system architecture.

Choreographing for the camera became a challenge early in the $20^{\text {th }}$ century as the motion picture industry evolved. Video-dance is now established, having moved from analog ancestors to digital successors, from Maya Deren's path-breaking A Study in Choreography for Camera (1945) to work of younger generations of video makers. At the 2005 Digital Cultures festival, a program of video-dance ('Motion at the Edge') persuasively reflected the aesthetic genres with which choreographer-filmmakers work today, ranging from the poetic to the documentary, the ethnographic to the abstractexperimental, plus various crossovers between video, dance and performance art. $^{2}$ All videos in the program featured images composed with very carefully choreographed camerawork enabling both a remoteness of settings and a deep intimacy of viewpoints rarely possible in theatre-based performances. When I attended the 2011 Cinedans Festival in Amsterdam, newer categories had been created - for example "One Minute Dances" and "Online Dances" - reflecting recent evolutions in the compression and uploading of dance to the internet. The prize for emerging filmmakers went to Fabian Kimoto’s The Rising Sun, a vivid kinetic portrait of a young hip hop company.

Beyond the screen-based medium, real-time interaction with camera-vision, sensor or artificial intelligence systems requires attention to the process patching, to larger issues of the space and sensory experience of the digital, as well as to constraint parameters. If dance is considered a medium, and if we look for artistically challenging 
dance content created by interactional choreography (immersively or with instruments, wearables, smart garments, body-worn technologies), an interactive-medium-specific analysis requires the examination of choreography, spatial design, and dancing with software patches in their own particular interactional manifestations. The digital, at the same time, is now being perceived more clearly as our contemporary phenomenological dimension, our technically mediated interface with 'mixed reality' (Hansen 2006: 5) as a social-media world - expanding to YouTube and the so-called 'New Aesthetics' of ephemeral curation in the blogs, Facebook, image boards, and tumblrs of net.culture. Future dance makers and performance artists will be 'born digital' and have grown up with pervasive computing, wearable smart technologies and a torrent of images.

\section{Tools and Choreographic Systems}

If we ask what models of interaction influenced the choreographic imagination recording and capture tools of course played a role as much as the electronic instruments and controllers used by musicians. Tools are enabling especially when they alter or constrain our approaches to performance making, and thus also invite reflection on our methods. Interactional artworks require the user (as in all participatory scenarios) to learn the rules of the system, intuit ways in which the system responds, testing various

potentials of systemic feedback when generating forms and sounds. In the early years of such interaction art, while there was much shadow play there was also real discovery of expressive physical, sensuous experience. Since bodies constantly change, in and through their ongoing relationships, a sentient environment can inspire dynamic bodily inscriptions, as forms, matters and sensations unfold. 
I remember the impact derived from Improvisation Technologies, a tool for the analytical dance eye released by well-known choreographer William Forsythe in 1999, with some 60 video chapters and animations in which he demonstrates essential principles of his motional language. ${ }^{3}$ An algorithmic approach to movement generation here at the same time becomes a sensible conceptual framework through an interactive training installation. Expanding on this idea, one could argue that all interactional systems imply particular articulations in continuously emergent relations. Forsythe has also created a major online research project, Synchronous Objects, which presents collaborative research on organizational principles in the choreography (conducted at Ohio State University's Advanced Computing Center for the Arts and Design). The researchers analyze and creatively redeploy spatial data from the dance (the case study is Forsythe's One Flat Thing, reproduced), re-visualizing the kinetic dispositif. Thus, Synchronous Objects becomes a series of re-mappings of the distributed flows of the dancers' movements providing tools that allow the user to trace, re-imagine and re-draw spatio-temporal behaviors from the dance (http://synchronousobjects.osu.edu).

The Croatian BADco. has also released a tool kit, Whatever Dance Toolbox (software by Daniel Turing), enabling image analysis of the process of compositional, improvisational and dynamic decision making, as well as the study of how a machine 'sees' performance and how we can think about the totality of relations between performer, system setup, choreography and generated data-outputs (images, sounds).

The Brasilian Cena 11 company, directed by Alejandro Ahmed, is another case of a group working directly with programmers developing their own custom-built systems. When I met them in 2007 they explored the combination of dance and robotics, similar to 
the experimentations of Margie Medlin (Quartet), Pablo Ventura (kubic's cube) or Garry Stewart's Australian Dance Theatre (Devolution). The dance \& technology community arguably grew over time because the initial custom-built systems were soon shared: Troika Ranch’s Mark Coniglio, who wrote the interactive software "Isadora” (available at www.troikaranch.org), demonstrated its application in innumerable workshops. International platforms such as IDAT, Digital Dance, Future Physical/bodydataspace, Digital Cultures, CYNETart, Boston Cyberarts or Monaco Dance Forum brought practitioners together for the exchange of knowledge and artistic methods.

The particular relationship of hybrid choreography to technology (for example Medlin's quartet for dancer, musician, and robots) illuminates the changing contexts for emergent performance concepts. They may be derived from new or hacked tools, or from innovative sci-art frameworks for the creation of digitally augmented human movement or digital motion archives. Forsythe's Synchronous Objects is a prominent case of such re-examination of digital technology in regard to its representations of the corporeal and of a choreographic system of operations that has implications for other biotechnological or design fields.

We must also keep historical precedents in perspective. Today's mocap-based animations, created in commercial film but also in computer science, human factors and graphic art departments that look for performers to be 'subjects' for capture, find their historical roots in late 19th century motion studies in chronophotography and early cinema. ${ }^{4}$ However, figure animation for avatar choreography today does not even need 
motion-capture systems and real subjects as it can just as easily be generated in software programs (Lifeforms, 3D Studio Max, Maya, etc).

Recent developments in sonic arts indicate that with the increase in computing power and available ProTools, software artists can more flexibly explore graphics in ways similar to experimental film practices. Real time synthesis and a kinaesthetic sensibility derived from motion graphics could contribute to the emergence of a new art based on structural aesthetic similarities between the two forms, similarities fundamental to an understanding of both experimental film and electronic music. Furthermore, supporting evidence from cognitive psychology and neuroscience (including new studies in sensorimotor perception, biological motion observation, movement dynamics and velocity, for example the "Choreography and Cognition" project initiated by Scott deLahunta) has generated renewed interest in structural approaches to artistic practice and perception. ${ }^{5}$

Finally, dance makers, researchers and teachers have used film/video as a vital means of documenting or analyzing existing choreographies that have been passed on, in practice, from performing bodies to other performing bodies. Some tools (LabanWriter, LifeForms, FIELD) attracted attention in the area of dance notation and preservation as well as among choreographers (for example Merce Cunningham, Pablo Ventura, Ivani Santana, Wayne McGregor) who wanted to utilize the computer for the invention and visualization of new movement possibilities. ${ }^{6}$ Computer engineers such as Frieder Weiss (who wrote the EyeCon software) are much sought-after collaborators - for example in Chunky Move’s Glow, a beautiful solo performed with interactive graphics projected 
onto the floor underneath the dancer whose movement becomes entangled with lines of light and cascading shapes. A video of Glow was quickly released and debated on the

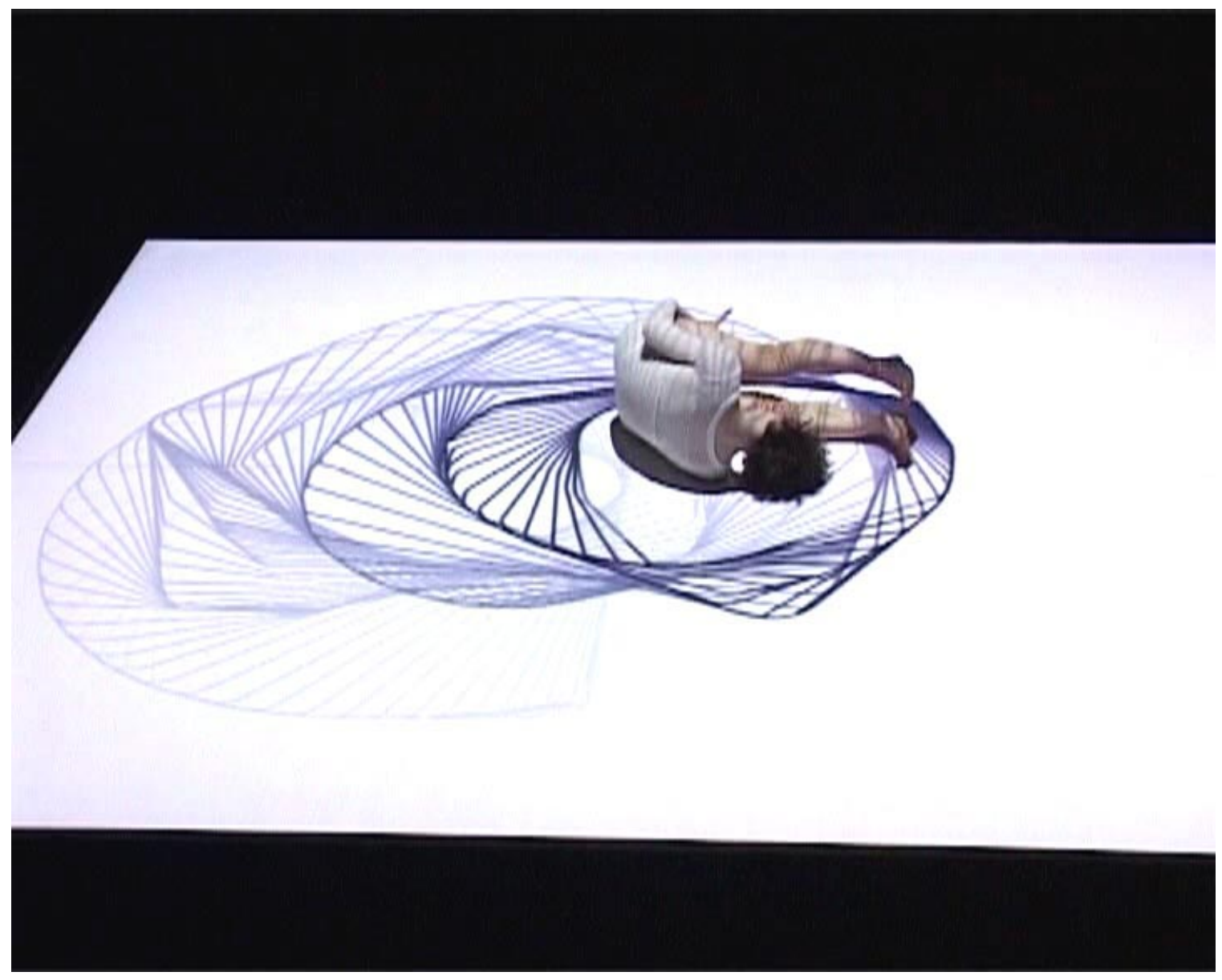

Photograph 30.2 Sara Black from Chunky Move in Glow (2007), choreography: Gideon Obarzanek, interface design by Frieder Weiss. Photo: Frieder Weiss.

dance-tech.net website, a thriving collective social network which embraces a crowdsourcing approach to document, discuss and disperse information on diverse practices and processes in the field, using a bottom-up method of collaboratively generated knowledge (in the manner of WIKIPEDIA). Chunky Moves' subsequent Mortal Engine production (2008), like Glow, was a particularly vivid example of a dance employing computer-vision and electronic-sensing techniques to augment the stage environment to 
an extent where moving bodies could hardly be distinguished from digital projections surrounding them. The system's technicity (in this case modeled after total surveillance, and thus potentially reprehensible) operates on continual analysis and processing of live data via real-time audio and video processing software, using downward projection to generate an ever-evolving audio-visual landscape. The corporeal movement is quantized by the software as the computer, of course, expects numerical data, not 'choreography'.

\section{Feedback Systems and Wearables}

This new writing of dance implies thresholding, scaling, filtering and parameterization, various processes that belong properly to computing/computer vision science, not aesthetics. What does the computer want? Frankly, I do not care so much anymore, and if this chapter would drift in a different direction, I should address the critical questions of gender, race and age, privilege and dis/ability that often get overlooked when 'technology' is spoken of as if it were neutral or a matter of functionality. Not drifting, I posit that techno-choreographic working methods incorporate instruments (cameras, data projectors, microphones, sensors, microcontrollers) and software tools allowing them to structure and control the various components of a performance event: sound, video, 3D animation, motion graphics, biofeedback, light. It is the convergence of choreography with instrument and system design - the languages of programming, electronic music and film editing in real-time processing - that I define here as ‘choreographic system’. We cannot look at human performers in the interface as separate from the interactive software system. In fact, software programs can also be performers of choreography. In 
the posthumanist sense, then, digital devices and algorithmic organizing have their own agency.

If we use a diachronic perspective, there are two generations of interactional design. In the first generation of interactive dance theatre of the 1990s, when 'mapping' (gesture to sound, gesture to video output) was explored in the interface configuration for performer and reactive environment, such understanding of the system was inspired by the cybernetic vision of feedback control and the modeling of the machine on the human actor. Direct interfaces (flex sensors, accelerometers, micro switches, pressure plates, and so on) required specific techniques of use which sometimes led choreographers to argue that the dancer acted as a live video editor or musical instrument. The dancer also often had to be wired up, and the wires showed openly. But aesthetic and conceptual concerns regarding the emergent techniques (criticized as limiting in their triggering function) eventually led to a search for alternate interfaces. Dance-tech or music-tech collaborations involving direct, gestural interfaces have declined even if some practitioners continue to argue that the interface should remain tangible so that mappings between performative input (gestural) and output (video/sonic) are easily inferred.

An analysis of specific artist-instrument combinations suggests that localized techniques had to be developed to combine choreography and improvisation; one can also identify a common set of software techniques (for example granular synthesis) and filtering parameters applied to digital video/sonic output. Especially with regard to digital dance/music collaborations on stage or in interactive installations, both the gestural and the software parameterization techniques should be given equal recognition. If there were a larger range of works available for analysis, one would be better able to distinguish the 
scales of sensor data values (able to be transformed by the reactive environment) from the particular performance technique - or through the style of choreographic improvisation harnessed for a particular output. It is not only the system that 'recognizes' gesture. The dancer also needs to sense, and develop somatic awareness, of how to move and express with a body-worn sensor or a Kinect camera looking on.

I shall evoke three performances. In July 2003, the gigantic Engine Room of the abandoned Coal Mine Göttelborn (Germany) was used to house a large crowd for an interactive sensor-dance, Titled On, which dramatized the dancer's breathing organism. The performance took place at the end of the first international Interaktionslabor. Upon entering a door to a staircase, the audience would glance down thirty or forty feet to an empty space where one of the two winding engines of ten thousand horsepower had stood, the remaining one now facing a gaping hole on the south side, the entire building a deep resonance body, with the western wall serving as film screen. The collaboration between Lynn Lukkas/Mark Henrickson (Minneapolis)/Paul Verity Smith(Bristol)/Marija Stamenkovic Herranz (Barcelona) and Kelli Dipple (Melbourne) opened up striking possibilities of the sensor-interface, pointing to 'spaces' in-between the aural, the rhythmic, the visual and the visceral.

\section{$<$ FIGURE 5 HERE >}

In this interface environment, the body's actions were measured not only as sound (via microphone) but as the most subtle variations in the biomechanics: the pulse, breath, and heart rhythm in the body itself (via a Bioradio attached with electrodes). The electrically 
measurable signals were transmitted wirelessly as data to the computer, where they affected not only the sound processes in real time but the rhythm of the image movement of the projected film sequences stored in the computer. Stamenkovic performed the dance of breath, first improvising softly with extended vocal techniques as she descended the staircase in midst of the audience, then purely with heavily amplified breathing as she moved onto the flat plane of the engine room, and finally with her whole body and staccato voice as she propelled herself into an untrammeled trance-like flurry of movement. Her voice crept under our skins, the magnificent resonating sound in the huge room entering through our pores, bones and stomachs. As we listened we realized how her breath controlled the image movement and thus the dramaturgy of the story. If Stamenkovic stopped her breath, the film's motion froze. When she breathed, we saw her (on film) walk across the slag heap of the Mine, descending into a hollow path. Lukkas had filmed her outside movement differently in each section, the third one using a hyperactive zoom. In conjunction with Stamenkovic’s accelerated breathing, this final segment materialized as pure hyperkinetic sensation, transforming the entire spacevolume into an irregular pulsating body-machine of continuously unfolding exhaustive yet libidinal intensities.

A performance of this kind is hard to describe. It produced an extended threedimensional space where pure sensation broke the continuity and stability of her own image (on film) even as she entered into a feedback loop with remembered movements she had enacted outside. Additional sound slowly grew inside the building, transforming sense perceptions of spatial images even further, or allowing the audience to recognize how their own sensations framed or pulsed the virtual images. Image-movement of 
landscape and figure, sound clusters and pebbles, breath and body, echoed and transformed one another in recursive couplings. Documentary photographs of such performances always disappoint. One sees a dancer and a screen projection, which in this case is nearly meaningless since all the other sensations and the volume of the space itself are lost. The sensorial coupling of interactivity and real time also derails the notion of a 'work' - there is no Titled On. It does not survive as a choreography, yet the technical diagram of the system awaits new concretizations. The performance event is entirely contingent on concrete situations, the interlaced process which produces itself in real time before a public.

Ten years onward, the Bioradio has disappeared into the costumes. In the DAPLab works I created with fashion designer Michèle Danjoux and our ensemble, we experiment with wearables/wearable space or what Danjoux calls 'design-in-motion'. Our installation UKIYO [Moveable Worlds] reflects long-term transcultural cooperation with artists/scientists in Japan and Singapore. Such colloborations are common for the encounter between choreography and science. Our system design for the installation architecture involves five hanamichi (runways) in the space, with the creation of analog and digital techniques for movement with sounding costumes on these territories. The wearables, each developed as particular characters, are crucial in the overall scenographic and choreographic organization of the real time interactive dance which also includes both asynchronous film worlds and synchronous virtual 3D worlds (Second Life).

UKIYO manifests the multifaceted, dynamic relational aspects of garments/accessories, performing bodies, and digital articulations happening in intimate 
spatial proximity to the audience moving inside/around the dance. Abandoning the exteriority of spectacle, this (post)choreographic system is immersive. It exemplifies the kind of sound-motion design we develop with DAP-Lab, looking carefully for extensions to earlier historical models (for example constructivism, silent film), also comparing Russian and Japanese inspirations for influencing the characters (Futurism, musique concrète, ukiyo-e prints, Kabuki theatre, manga, deconstructivist fashion). This method we also pursued in for the time being/Victory over the sun (2014). In our systems we mix old and new media, allowing performers to develop their movement vocabulary through the potentials and constraints of the wearables, the flow of materiality and visceral sensuality discovered in the nuances of inter-action with costumes. 


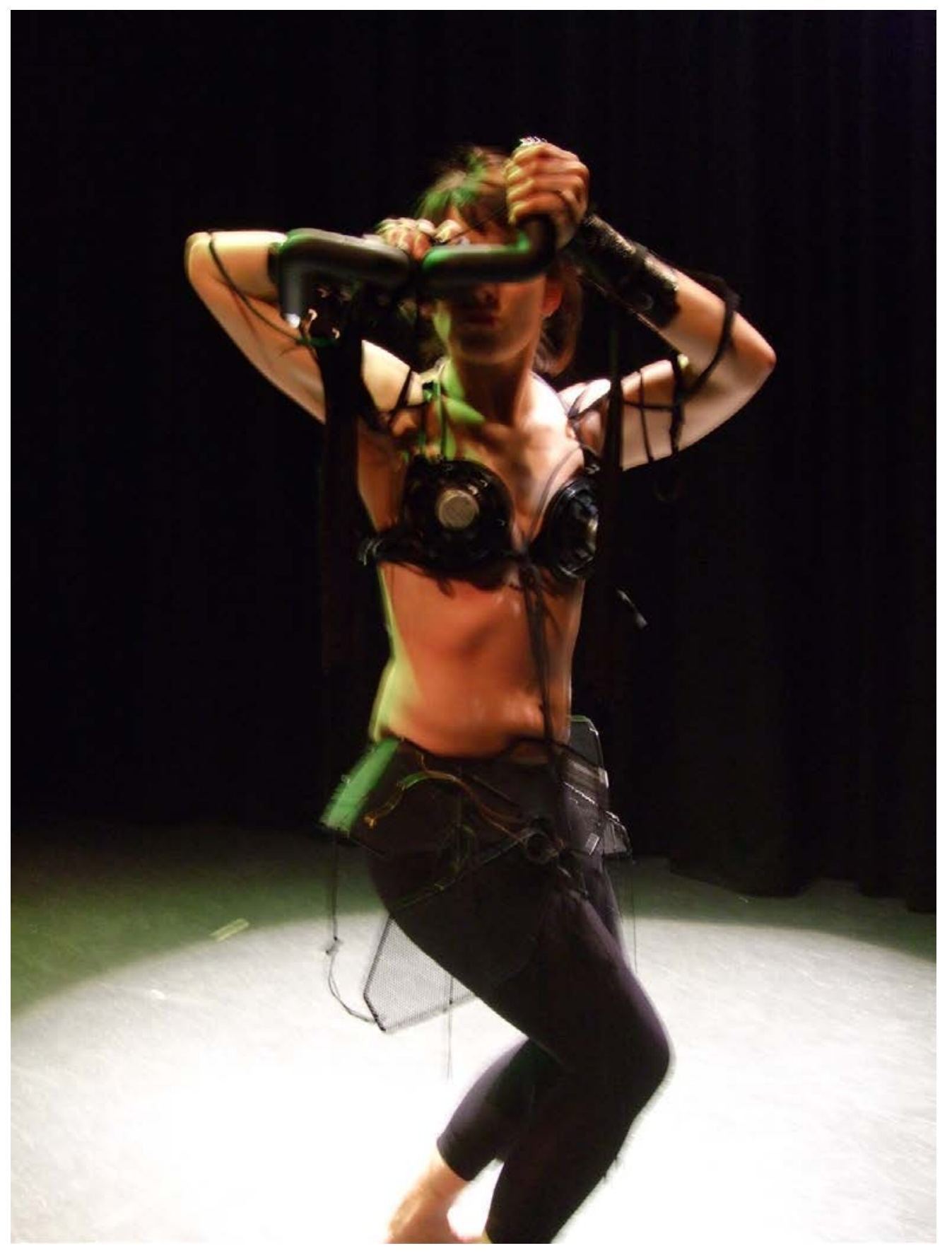

Photograph 30.3 Anne Laure Misme as WorkerWoman in UKIYO (2010), design by Michèle Danjoux. (C) Photo courtesy of DAP-Lab.

In $U K I Y O$, analog technologies were foregrounded, to an extent, in a performance fully embedded in a digital programming environment. Some of our wearable techniques use a 'cracked media' approach, for example the dancers actuate tools of media playback 
by distorting original functions as a simple playback device for prerecorded sound or image (see Fig. 6). Some of the costumes use dysfunctional audio objects, others translate musical instruments (such as the bandoneon) into hieratic garments evoking a surreal (Kyogen) character. Some of the movement language in Act II is 'learnt' and adopted, copying avatar choreography developed by software. Delineating the main kinaesonic features of the UKIYO system would stretch beyond the scope of this chapter, but I wanted to refer to wearable technologies as an important dimension of contemporary performance design (inspired by developments in fashion and smart textiles but also in nanotechnology and affective computing).

\section{Choreographic Systems and Artificial Intelligence}

My second example is neither site-contingent nor improvisatory but reflects interactive choreography which can be repeated and cued within the various states of the system. It might be contradictory to speak of repeatable choreography when introducing the second generation of interactive systems, since the continuity of computer processing co-evolves with the dance movement and generates its own creative behavior that might be readapted into the choreography. Whereas the first interactivity understood humancomputer interaction on a stimulus-response or action-reaction model, the second interactivity emphasizes sensorial dialogue insofar as human enaction and machinic processes each have their own autonomy, being able to self-reorganize in constant dynamic relationship.

Second-generation interactivity heightens the experience of human embodiment as the sensory coupling of dancer and virtual environment evolves in noncausal (non- 
triggering) correlation with one another. Ideally, both performer and performance system respond to the other's enaction by undergoing self-permutations on the basis of distinct operational rules (a form of 'post-choreography') which are internal to them. ${ }^{7}$ Moving towards indirect interfaces (optical, magnetic, and ultrasonic sensors or machine vision), however, creators of such performance systems often prioritize the development of software techniques over physical techniques. In innumerable performances of this kind one sees mediocre or underdeveloped dancing. In such cases, perfunctory physical techniques are used to patch the interface rather than expanding the transformational capabilities of the system or developing new re-organizations of the body and its expressive metabolism. The situation tends to be worse, I think, in interactive installations inviting an unprepared public to move around and become 'co-authors'.

In an indirect interface, the performers (or participants) are challenged to reorganize their motional, affective, perceptive and proprioceptive behavior in the environment. The desired aesthetic aim would be to anticipate direct dance transformations or disjunctive, differential relations in real time. Code and matter symbiotically differentiate, alien perspectives open up, unholy alliances emerge when strange avatars appear 'face-to-face'. In other words, the more complex the technologies behind the interface become, the more attention, creativity and originality need to be applied to transformative techniques and (dis)synaesthetic processes.

A first response to this challenge can be observed today in the care given to the subtleties and nuances of gestural quality. Troika Ranch's piece 16 (R)evolutions is a dance which almost reads as an allegory of the evolutionary development of gestural control and refinement of motion tracking within the programming environment they 
have created. Rather than deploying a high-end multi-camera motion capture system for real time graphic animation, as it was used in Trisha Brown's how long does the subject linger on the edge of the volume... (2005), Troika Ranch designed a small system that can easily travel and is inexpensive, combining a small camera with Isadora and a motion capture software (Eyesweb) created by Italian scientists at the Genova InfoMus Lab that allows a particular gesture analysis here used for the transformation of points in space, contours, lines, and motion energy or direction into animated graphics. It is tempting to call this a process of real time translation. The dancers onstage move and the system analyses the motion by generating graphic shapes in the digital screen projection. In this computational environment, movement-action and motion graphics co-evolve. The points in space, recognized each second almost as in Marey's or Muybridge's chronophotography a hundred years ago, are here transformed instantly in real-time. They generate a trail of successive movements in fluid continuity which form a Gestalt or, rather, Gestalten (in the plural). 


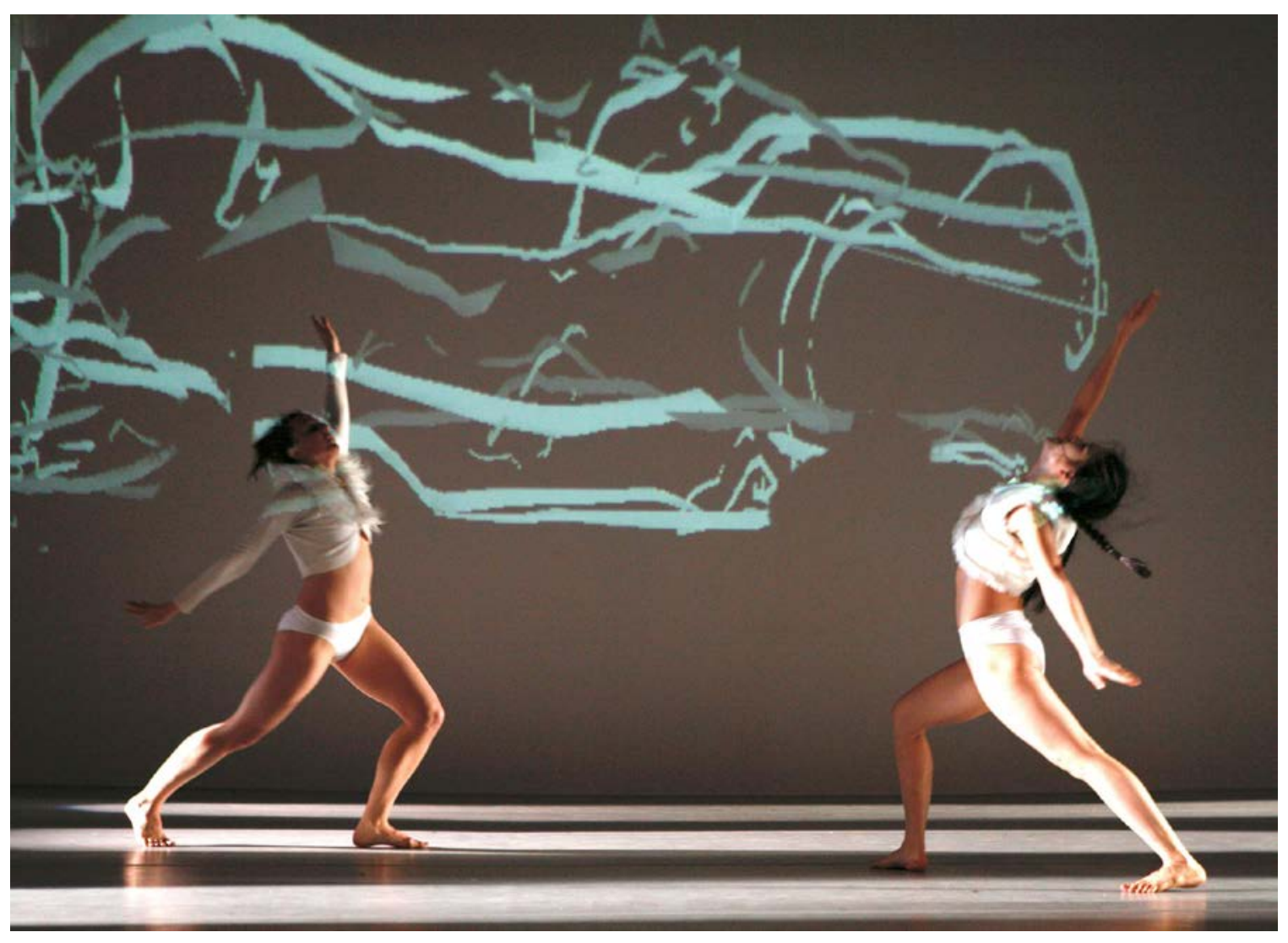

Photograph 30.4 Johanna Levy and Lucia Tong dancing in Troika Ranch's 16 (R)evolutions (2005). Photo: Richard Termine, courtesy Mark Coniglio.

At this juncture in the development of real-time motion tracking, the interactivity is no longer focused on direct mapping of gesture but on the creation of complex 'action paintings' or action palimpsests, calligraphies of human gesture translated into imageflows. In Performance, Technology \& Science (2009: 155-156) I compare the digital system at work here with the extraordinary analog dance with calligraphies created in Cloud Gate's Cursive (2006). Cloud Gate’s flowing ink is real, and the dancers move in front of the suspended rice paper panels; the trails of energy mingle. Troika Ranch's virtual calligraphies emerge projected from an interface which is opaque. Mathematically, the procedure has a clear grammar: Isadora tracks motion and analyzes 
the numeric data. The software functions as a measuring tool or tool of observation. Depending on the values, filters and modifiers assigned to the data, the program analyzes slight changes in the motion gesture - observing the 'living state' or properties of such movement (four categories: straight, curved, lateral, complex). Recognizing change of direction, speed, dynamics and velocity of movement within these categories, the program then renders the graphic output in real-time, and we can perceive the threedimensional dance and the projected 3D worlds of colors and shapes. Using a musical analogy, one could argue that the software program observes "tonal" qualities of the dancer's movement.

Another level of critical analysis could then be applied to particular choices the designers make for the visualization of data and feedback qualities of the control system. Numerous researchers in the hard sciences, including molecular biologists working on cellular dynamics and chemical transformations, are currently preoccupied with visualization technologies, and here an exchange of knowledge between fields of observation appears relevant, even if aesthetic or political questions about the meaning and affect of gestures may address different concerns from those of the cell biologist. Yet artists and cell biologists both show concern for pattern recognition and micro-behavioral change. It is worth dwelling on such procedures with which we construct categories for observation.

The computer, for example, cannot 'feel' the gesture in the way in which the human audience will sense the weight or import of a particular movement behavior and quality of expression. Coniglio admits he would not know himself where a gesture begins and ends, where it 'divides'. ${ }^{8}$ His software reacts to properties of the motion and is set to 
modify the Gestalt of the image we see projected continuously (color changes; change in size; rotating planes to effect a more three-dimensional and topsy-turvy feeling of the images). The images themselves can have various tactile characteristics connected to the 'gestural-ness' of drawing and painting (sinewy) or the more architectural look of geometric, polygonal shapes (rigid). In one scene of 16 (R)evolutions, a meshwork of lines (vertical and horizontal) appears all over the floor and back projection which is pulsating and constantly moving, growing, decreasing, turning, evolving. In another, a meshwork of more densified criss-crossed lines and architectural Gestalts gains polyphonic complexity in motion, and in rotations that defy Euclidian space.

Choreographer Dawn Stoppiello suggests that such explorations in motion tracking and visualization emphasize highly subtle manipulations of visual and aural qualities, correlated to new concepts of dynamic or semi-chaotic systems whose philosophical and scientific thought-models are derived from research in biology, a-life, computer science and cognitive science (Maturana/Varela, Prigogine/Stengers, Kauffman, Iberall, and others). No longer based on notational systems (Labanotation) but on computational analysis and mathematics, 'description of movement' is rendered as image-movement, yet the fuzzy logic in the chaotic state of the system reminds us how difficult it is to speak of a digital aesthetics. The digital medium itself is indifferent to movement poetics or authorship.

Dance and interaction designers, in other words, now reflect on what could be called the psychology of spontaneous, intuitive, unpredictable or ritualized behavior in 'traversable interfaces' which allow fluid transitions between digitally augmented human/machinic movement. Nicolás Salazar Sutil (2015) speaks of kinetopoiesis, based 
on mathematically formalized movement. The difference to earlier anthropological studies of role behavior lies in the fact that performance is here always understood to take place in relationship to system-design which often embeds performer and interface within a physically traversable projected display or immersive environment, and thus within the time/duration of virtual abstractions as it was demonstrated in Cunningham's BIPED. When robots and avatars are involved, the language of object manipulation (actuators) enters the scene, creating a fascinating, complex re-orientation of our old anthropocentric assumptions about presence and agency.

Trisha Brown's stage work, how long does the subject linger on the edge of the volume..., recreated for the 2006 Monaco Dance Forum, interfaces with animated graphics from real-time motion-capture driven by a custom-built artificial-intelligence software that responds to the kinematic data and generates particular behaviors. Marc Downie speaks of 'choreographing' these extended agent-bodies, but he carefully distinguishes such motion behavior from human, physical intelligence. The software draws its own dance diagrams live during the performance, and the graphic agents are projected on a transparent scrim in front of the stage. The agents are software 'creatures', acting according to their artificial intelligence. They have their own autonomy. Their imagery comes about as they picture things to themselves, trying to make sense of what they sense onstage in real-time as the dance unfolds.

For example, how long opens up with a triangle-creature, whose intention is to move from stage right to stage left. It does so by hitching rides on points in the motioncaptured dancers' bodies, guessing which ones are moving in the right direction. It extends a line out to a likely point, and is then tugged that way if it has guessed correctly. 
Sometimes its hunch is wrong; it has to relinquish its grip on that point and await the next opportunity. In such a case, that line is left as a trace, and thus the whole image as it progresses is simultaneously a history of its attempts. This virtual 'choreography', in other words, has memory.

The Monaco Dance Festival testified to such surprising advances in digital composition, as we watched the physical intelligence of Brown's dancers interact with the artificial intelligence of Downie and Kaiser's 'thinking images'. In a workshop, Downie emphasized that the computer is an embodied agent, deeply coupled to its environment such that its actions on its environment - mediated by the physical constraints of some virtual animated body - must be carefully produced and its perceptions of its environment - mediated by its limited sensory apparatus - must be carefully maintained. The machine is learning from dance; it can be trained to do so. The creatures' bodies and their physics are purely imaginary, of course, and it is noteworthy that the software artists prefer indeterminate images, lingering between abstraction and figuration, hinting perhaps at the spiritual in art once described by Kandinsky during his teaching at the Bauhaus. Many of today's dance-tech workshops and projects continue the great modernist tradition of Kandinsky's painting and Schlemmer's Bauhaus dances, to which my own work with the DAP-Lab is also indebted.

The maintenance of the motion analysis and real-time rendering system for how long involved a huge technical effort, unlikely to be repeated too often on the choreographer's busy touring schedule. Such work is also built on extensive research involving numerous artists and scientists over a period of years: laboratory conditions not generally available to dance companies. I am aware that these are slowly being created at 
universities or art labs across the world. Young artists find ways to build their own collaborative networks, use unconventional approaches (reverse engineering, adapting game engines or mobile devices) to make use of media assemblages in performance. It is important to acknowledge the diversity of interactional possibilities. Yet it is also crucial that dancers and designers have sustained time to find and develop specific performer techniques which can be trained. Each dance or media festival thus also provides an occasion to encourage critical dialogue between pioneers and newcomers, artists, audiences and scholars, in order to foster knowledge transfer for placing and evaluating new methods of practice and the provocative resonance of the systems and avatarperformer connections that stretch familiar frames of reference.

${ }^{1}$ The most comprehensive study of new technological performance is found in Dixon (2007). See also Birringer 2009, Salter 2010, Kwastek 2013.

${ }^{2}$ See: http://www.digitalcultures.org/Exhibits/motion.html.

${ }^{3}$ See Birringer 2002. Emio Greco/PC’s installation Double Skin/Double Mind (2007), developed with Bertha Bermúdez, attempted something similar.

${ }^{4}$ For critical discussions of dance technology and motion studies, see Reilly 2013, Salazar Sutil and Popat 2015, Salazar Sutil 2015.

${ }^{5}$ For information on this research project, see http://www.choreocog.net. For current crossovers between neuroscience and dance, see Birringer and Fenger 2005, Reynolds and Reason 2012.

${ }^{6}$ Software systems used in dance include: VNS, BigEye, Image/ine, EyeCon, Kalypso, Max/Msp/Jitter, Nato, ChoreoGraph, EyesWeb, Isadora, Keystroke, dance Forms, and 
numerous VJ'ing tools. PD (Pure Data) is an open source alternative to Max/Msp. An overview of software development for performance is offered by Scott deLahunta: $<$ http://www.sdela.dds.nl//transdance/report/>. He organized the path-breaking workshop “Software for Dancers” at Sadler's Wells in London (2001). We followed it up with "Performance Tools: Dance and Interactive Systems" at OSU in 2002. He then coordinated the research project "Choreographic Objects: traces and artifacts of physical intelligence”, which includes Forsythe's Synchronous Objects, Emio Greco/PC’s Capturing Intention, Wayne McGregor's Autonomous Choreographic Agents, and the Siobhan Davies Dance Archive. Scott deLahunta leads the Motion Bank Project (Forsythe Company): http://motionbank.org. For architectural systems theory, see Spuybroek 2004.

${ }^{7}$ I began to theorize the 'post-choreographic' in 2006 (http://interaktionslabor.de), and in later publications (Birringer 2008), implying that systems alter our control over creation. For videos of recent DAP-Lab works, see http://youtu.be/WeAIYCnsDe4, and https://youtu.be/g2yfYrlvOLM.

8 An early version of 16 (R)evolutions was shown at Digital Cultures (2005): http://www.digitalcultures.org. Coniglio and Stoppiello commented on their work in a post-show discussion. For research into kinaesthetic empathy, conducted by dance practitioners/cognitive scientists, see “Watching Dance”: http://www.watchingdance.org/.

\section{References}

Birringer, Johannes (2002) ‘Algorithms for Movement’, PAJ, 7 (1): 115-119.

----- (2008) ‘After Choreography’, Performance Research, 13 (1): 118-122. 
----- (2009) Performance, Technology, and Science, New York: PAJ Publications.

Birringer, Johannes and Fenger, Josephine (eds.) (2005) Tanz im Kopf/Dance and Cognition, Münster: LIT Verlag.

Dixon, Steve (2007) Digital Performance: A History of New Media in Theater, Dance, Performance Art and Installation, Cambridge, MA.: MIT Press.

Giannachi, Gabriella (2004) Virtual Theatres: An Introduction, London: Routledge. Hansen, Mark B.N. (2006) Bodies in Code: Interfaces with Digital Media, New York: Routledge.

Kwastek, Katja (2013) Aesthetics of Interaction in Digital Art, Cambridge, MA.: MIT Press.

Parker-Starbuck, Jennifer (2011) Cyborg Theatre: Corporeal/Technological Intersections in Multimedia Performance, Basingstoke: Palgrave Macmillan.

Reilly, Kara (ed,) (2013) Theatre, Performance, and Analogue Technology: Historical Interfaces and Intermedialities, Basingstoke: Palgrave Macmillan.

Reynolds, Dee and Reason, Matthew (eds.) (2012) Kinesthetic Empathy in Creative and Cultural Practices, Bristol: Intellect.

Salazar Sutil, Nicolás (2015) Motion and Representation, Cambridge, MA: MIT Press.

Salazar Sutil, Nicolás and Sita Popat (eds,) (2015) Digital Movement: Essays in Motion Technology and Performance, London: Palgrave Macmillan.

Salter, Chris (2010) Entangled: Technology and the Transformation of Performance, Cambridge, MA: MIT Press.

Spuybroek, Lars (2004) NOX: Machining Architecture, London: Thames \& Hudson. 